\title{
Fatores determinantes da evasão universitária: uma análise com alunos da UNISINOS
}

\author{
Vanessa Viégas Alves Furtado \\ Bacharel em Ciências Econômicas \\ Mestre em Economia (UNISINOS) \\ vivavan2002@yahoo.com.br
}

\author{
Tiago Wickstrom Alves \\ Doutor em Economia \\ Professor do Mestrado em Economia da UNISINOS \\ twa@unisinos.br,wickstrom.alves@gmail.com
}

ISSNe 2178-9258

ISSN 1678-2089

Organização: Comitê Científico Interinstitucional

Editor Científico: José Carlos Lázaro

Avaliação: Double Blind Review pelo SEER/OJS

Revisão: Gramatical, normativa e de formatação

Recebido em 13-03-2010

Aprovação Final em 02-12-2012

\section{RESUMO:}

O presente trabalho tem como objetivo realizar uma análise dos fatores determinantes da evasão universitária na Universidade do Vale do Rio dos Sinos (UNISINOS). O estudo foi conduzido com base em uma amostra representativa de alunos da UNISINOS, ingressantes no período de 2005 a 2008, tendo avaliado, por meio de regressão Logit, a relação entre variáveis socioeconômicas e evasão discente. Pelos resultados da pesquisa concluiu-se que algumas variáveis pesquisadas possuíam relação crescente à evasão, como, por exemplo, a variável disciplinas canceladas, em que um número maior de cancelamentos resultou em uma probabilidade mais elevada de evasão por parte dos alunos. Outras variáveis possuíam relação inversa a esse fenômeno, como a variável disciplinas cursadas, na qual um número maior de disciplinas cursadas por semestre reduzia as chances de o estudante evadir.

Palavras-chave: Evasão universitária. Regressão Logit. Determinantes da evasão. Custo de oportunidade. Gestão universitária.

\section{ABSTRACT:}

This work aims to conduct an analysis of the determinants of university dropout from the University of the Bells River Valley (UNISINOS). The study was conducted from a representative sample of students in UNISINOS, entrants in the period from 2005 to 2008, and evaluated by Logit regression of the relationship between socioeconomic variables and dropout students. From the search results it was concluded that some of the variables investigated had increased relative to the avoidance, for example, the variable subjects canceled, wherein a greater number of cancellations resulted in a higher probability of escaping from the students. Like others who had inverse relation to this phenomenon, as the variable subjects taken, in which a greater number of courses taken per semester, reduced the chances of evading student.

Keywords: Dropout of university. Logit regression. Determinant of dropout of university. Opportunity cost. University administration. 


\section{INTRODUÇÃO}

A evasão universitária é um fenômeno cada vez mais comum em universidades privadas de todo o mundo, independentemente das peculiaridades socioeconômicas e culturais de cada país e das diferenças entre as diversas instituições de ensino. A África do Sul, por exemplo, apresenta uma taxa de $40 \%$ de abandono dos estudantes universitários no primeiro ano de graduação. De acordo com Macgregor (2007), as principais causas desse abandono seriam as dificuldades financeiras enfrentadas pelos alunos, além da baixa escolaridade e renda da família na qual estão inseridos.

Já no continente europeu, conforme Garner (2007), a Irlanda apresenta a menor taxa de evasão universitária, seguida pela Inglaterra. Em termos mundiais, o país com a menor taxa de evasão é o Japão. É interessante saber que uma das maiores taxas de abandono entre os países desenvolvidos está nos Estados Unidos, onde apenas aproximadamente $50 \%$ dos jovens dão continuidade ao ensino superior, uma vez que as faculdades naquele país permitem uma ampla flexibilidade na formação, o que deveria minimizar o abandono pelo efeito da opção errada pelo curso.

No Brasil, segundo Almeida e Veloso (2002), em alguns casos, o baixo desempenho no ensino médio reflete no desempenho das primeiras disciplinas do curso superior, resultando em abandono do curso pelas reprovações nos primeiros semestres. Outro fator seria o fato de o aluno buscar o curso de baixa demanda com o objetivo de, após ter ingressado, procurar o curso de sua verdadeira opção através da transferência interna. Como isso às vezes não é viabilizado, principalmente pela não oferta de vaga para esse tipo de ingresso, o aluno busca um novo concurso vestibular em outra instituição.

Nassar et al. (2003) afirmam que a evasão universitária no Brasil apresenta um índice muito elevado, cerca de $40 \%$, o que leva a crer que as Instituições de Ensino Superior (IES) não estão se ocupando adequadamente desse fenômeno, não só em termos de gestão dos cursos já existentes como de abertura de novos cursos. Para Vieira e Frigo (1991), a principal causa da evasão poderia ser a reforma do antigo ensino de $2^{\circ}$ grau, que viabilizou o ingresso precoce de muitos jovens nas universidades, o que os levou à escolha prematura de um curso, quando ainda desconheciam todo o potencial de suas aptidões, motivações e de seus interesses. Outra causa apontada por Vieira e Frigo (1991) é o fato de muitos universitários serem "trabalhadores-estudantes", em que a dificuldade de conciliar o trabalho - meio necessário para a subsistência - com os estudos muitas vezes se torna inviável.

Outra percepção interessante a respeito da evasão surge do trabalho de Ristoff(1997). Para esse autor, a evasão nas universidades do Brasil surge no âmbito de duas grandes crises que se vive no setor, a de modelo e a gerencial, uma vez que ambas apontam para o descomprometimento gradual do Estado com a educação.

Abordando por outro prisma, Silva (2006) indica que a magnitude da evasão está diretamente relacionada com a qualificação do corpo docente e não somente com o status socioeconômico dos estudantes. Para ele, os jovens amadurecem mais tarde, fazendo algumas vezes escolhas prematuras no que diz respeito ao seu futuro profissional, e muitos deles sofrem da síndrome da adolescência prolongada, revendo os seus objetivos de vida por volta dos trinta anos.

A pesquisa desenvolvida por Theóphilo e Moraes (2005) complementa a de Silva ao revelar que a evasão no início do curso estaria, normalmente, relacionada à dificuldade do aluno em se adaptar às exigências dos professores e à mudança do ensino médio para o superior. Já quando os alunos evadem por volta do quarto e do sexto semestres geralmente é porque começaram a se questionar sobre o sentido da profissão (THEÓPHILO; MORAES, 2005).

No entanto, Biazus (2004 apud SILVA, 2006) afirma que os fatores da evasão discente se manifestam em graus distintos nos mais variados cursos das IES e que não há uma lógica uniforme que explique a homogeneidade da ocorrência no conjunto dos cursos, uma vez que esses fatores estão relacionados a: i) características individuais, como a vocação e outros problemas de ordem pessoal; ii) elementos internos, referentes aos recursos humanos, aos aspectos didático-pedagógicos e à infraestrutura; iii) elementos externos às Instituições de Ensino Superior, ligados a aspectos sociopolítico-econômicos.

Acredita-se, porém, que existam elementos comuns que induzam os alunos a abandonarem os cursos de graduação. Diversos desses elementos foram destacados pelos autores citados anteriormente e, embora se considere a crítica de Biazus (2004 apud SILVA, 2006), o que se busca neste trabalho é responder, com uma pesquisa exploratória, a seguinte questão: quais são os fatores relevantes da evasão dos alunos da UNISINOS? O objetivo específico deste trabalho é, pois, definir quais são as condições determinantes da evasão dos alunos da UNISINOS.

Embora não se possa generalizar para os estudantes universitários brasileiros, pois a análise foi realizada com alunos de uma universidade, destaca-se que este estudo é um dos mais amplos realizados por duas razões. A primeira reside no fato de que ele não cobre apenas um determinado curso, como fazem os estudos que têm sido realizados em sua expressiva maioria, mas sim 
contempla os 65 cursos de graduação da UNISINOS. A segunda razão se refere ao elevado número de variáveis socioeconômicas utilizadas na análise.

Em síntese, a evasão universitária é uma problemática que tem levado muitos estudiosos a pesquisarem as suas causas, a fim de que possam ser encontradas alternativas para as mazelas que se originam desse processo. E, apesar das concordâncias e divergências entre os autores, todos opinam igualmente que as universidades devem adotar processos de gestão que sejam corretivos e preventivos da evasão, reduzindo assim os índices de abandono. E, para tal, é fundamental a compreensão dos fatores que condicionam a evasão.

O projeto foi desenvolvido na UNISINOS e a análise partiu da elaboração e estimação de um modelo de regressão logística, estabelecendo a relação entre a variável qualitativa permanência/evasão dos estudantes dessa universidade com variáveis econômicas e sociais dos alunos. É importante destacar que o conceito de evasão utilizado nesta pesquisa foi o de Pereira (1995), que a define como aquela em que o aluno deixa a universidade sem concluir nenhum curso, o que excluiria a opção de mobilidade interna, mas não a mobilidade entre instituições.

A apresentação dos resultados da investigação está estruturada em três capítulos além desta introdução e do referencial teórico. O capítulo 3 descreve os procedimentos metodológicos adotados para a estimação do modelo logístico. No capítulo 4, desenvolve-se a análise e interpretação dos dados e, por fim, no capítulo 5 , apresentam-se as considerações finais.

\section{REVISÃO TEÓRICA}

A evasão universitária se ampliou no Brasil na última década e fez com que os gestores dessas instituições, fossem elas públicas ou privadas, definissem como meta o combate a essa evasão (BORGES, 2012). Esse fenômeno tem ocorrido em nível mundial e tem despertado o interesse de uma ampla gama de pesquisadores de diferentes áreas (BARDAGI e HUTZ, 2005) e a “[...] busca de suas causas tem sido objeto de muitos trabalhos e pesquisas educacionais" (SILVA FILHO et al., 2007).

A procura pelas causas ou pelos determinantes da evasão tem sido realizada por uma extensa série de métodos. Alguns deles avaliam com base em dados específicos de uma instituição, o que permite uma riqueza de detalhes para testes estatísticos ou para avaliações qualitativas, com base em entrevistas com os alunos, como no caso da pesquisa desenvolvida por Theóphilo e Moraes (2005). Outros trabalhos fazem uma abordagem macro, com base em censos, como o caso da pesquisa de Silva Filho et al.
(2007).

Contudo, independentemente de ser uma abordagem micro ou macro, quando há disponibilidade de dados, a técnica estatística amplamente utilizada e que tem apresentado resultados importantes é a análise de regressão com a variável dependente binária, ou seja, modelos econométricos probabilísticos. São exemplos de estudos que se utilizaram dessa técnica a pesquisa desenvolvida por Deke (2003), que associou a evasão com a qualidade da educação; o trabalho de Vitelli (2012), que buscou explicar os fatores que interferiam na probabilidade de evasão dos alunos; o estudo de Stratton (2008), que analisou se havia diferença entre aqueles alunos que abandonavam no primeiro ano e os que trancavam o curso por um curto tempo.

Os estudos nessa área convergem para alguns fatores determinantes da evasão, como: i) capacidade financeira dos alunos - Macgregor (2007) e Vieira e Frigo (1991); ii) taxa de reprovação nas primeiras disciplinas do curso - Almeida e Veloso (2002) e Theóphilo e Moraes (2005); iii) complexidade do mercado de trabalho e idade precoce para decisão do curso universitário por parte dos jovens Vieira e Frigo (1991). De fato, esses elementos podem ser resumidos em fatores acadêmicos, econômicos, sociais e emocionais, conforme observado por Majzub e Rais (2010).

Por fim, não se poderia deixar de destacar que há uma verdade intrínseca na afirmação da Baggi e Lopes (2011) de que "[...] a evasão escolar no ensino superior é um fenômeno complexo e, portanto, não pode ser analisado fora de um contexto histórico mais amplo, pois é reflexo da realidade de níveis anteriores de ensino, influenciando de diversas maneiras para o abandono de um curso superior".

\section{ASPECTOS METODOLÓGICOS}

O método estatístico utilizado nesta pesquisa é a regressão logística, que permite estabelecer os determinantes da evasão, conforme o objetivo do trabalho, e que tem sido amplamente utilizado em estudos que abordam essa temática.

O software utilizado para estimar os parâmetros da regressão foi o Eviews 7 e as informações vieram de fontes primárias disponibilizadas pela UNISINOS referentes ao questionário aplicado aos vestibulandos e de dados da base de registro acadêmico dos alunos. Esses dados serviram de apoio para a construção do modelo Logit, aplicado em uma amostra de 14.755 alunos que ingressaram por vestibular na universidade no período de 2005 a 2008, cujo modelo é:

$$
P_{j}=\frac{1}{1+e^{-\left(\beta_{1}+\Sigma_{i} \beta_{i} X_{i}\right)}}
$$


Onde $\boldsymbol{P}_{j}$ é a probabilidade de o indivíduo “j” evadir da universidade, i é a variável explicativa definida como i: i $\epsilon[1,190], \boldsymbol{\beta}$ é o vetor coluna dos parâmetros a serem estimados de ordem (190 x 1), $\boldsymbol{X}_{i}$ é a matriz contendo as variáveis explicativas a serem exploradas no modelo. A lista detalhada dessas variáveis encontra-se no Apêndice.

Destaca-se que a constante foi definida como mulher; que não informou o estado civil; moradora de São Leopoldo e que não participou do Programa de Ingresso ao Ensino Superior (PEIES); que não prestou o Exame Nacional do Ensino Médio (ENEM); que não informou o grau de instrução do pai nem o da mãe; que não informou o tipo de ensino médio concluído nem o tipo de escola e nem o turno em que concluiu o ensino médio; que frequentou o curso pré-vestibular; que não fez vestibular em semestres anteriores e o está fazendo apenas na UNISINOS; que nunca iniciou algum curso superior e o motivo principal da escolha do curso para o qual fez o vestibular foi outro, não constante nas opções relacionadas no questionário aplicado aos vestibulandos; que não exercia nenhum tipo de atividade remunerada; que não participava da vida econômica da família, pois não trabalhava; que tinha renda familiar situada entre vinte salários mínimos ou mais; que cursava Administração com Habilitação em Administração de Empresas Noturno.

Ainda, foram extraídos na análise os cursos de Administração com Habilitação em Administração Hospitalar, Bacharel em Sistemas de Informação, Filosofia Bacharelado, Licenciatura em Pedagogia Diurno, Formação de Escritores e Agentes Literários Noturno, Formação de Escritores e Agentes Literários e Tecnólogo em Gestão Cultural. Esses cursos tinham como característica o fato de serem novos ou estarem em extinção na universidade ou, ainda, possuírem basicamente 0 (zero) ou 1 (um) na totalidade dos alunos que constavam na base de dados. Destaca-se que se trabalhou com o total de alunos da UNISINOS que ingressaram no período de 2005 a 2008.

O modelo da equação (1) foi o de estimação original que incluía todas as variáveis. Após a estimação dele, passou-se a avaliar o nível de significância e pelo teste da Razão de Máxima Verossimilhança pertinência ou não da variável. Detectada uma variável redundante, ela era retirada e novamente estimada. Optou-se por esse procedimento, em oposição ao de stepwise, para que se pudesse avaliar o comportamento das variáveis em cada etapa, de modo que fosse possível ampliar o entendimento do comportamento delas.

\section{ANÁLISE E INTERPRETAÇÃO DOS RESULTADOS}

Este capítulo inicia com uma análise descritiva das variáveis que compuseram o modelo e continua, na seção seguinte, com a análise do modelo logístico, a partir do qual foi possível estabelecer alguns dos fatores determinantes que levaram os alunos da UNISINOS ingressantes no período de 2005 a 2008 a evadir.

\subsection{Análise Descritiva dos Dados}

Avaliando os dados da amostra (14.755 alunos), podese constatar que ela era composta por $52,28 \%$ do sexo feminino (7.714) e $47,72 \%$ do sexo masculino. Do total dos alunos pesquisados, $7.279(49,33 \%)$ permaneceram ativos enquanto $50,67 \%$ evadiram. Esse dado revela a magnitude da evasão nessa universidade, que, em apenas quatro anos, metade dos alunos, aproximadamente, evadiram.

Em termos de ingresso, constatou-se que os maiores índices ocorreram nos primeiros semestres de cada ano, uma vez que no segundo semestre letivo as matrículas se reduziam quase pela metade. O maior número de calouros foi observado no primeiro semestre do ano de 2008, que apresentou uma taxa de $22,09 \%$, ou seja, mais de $1 / 5$ de todas as matrículas realizadas no período de análise. Por sua vez, o segundo semestre de 2007 foi o que apresentou o menor número de matrículas, com uma taxa de apenas $8,71 \%$ sobre o total.

Já no que diz respeito à cidade em que residem os universitários da UNISINOS, verificou-se que a maior participação é da cidade de São Leopoldo, município onde se localiza a universidade, conforme se pode constatar na Tabela 1. 
Fatores determinantes da evasão universitária: uma análise com alunos da UNISINOS

Tabela 1 - Municípios em que residem os alunos da
UNISINOS ingressantes no período de 2005 a 2008
\begin{tabular}{|lcllcc|}
\hline \multicolumn{1}{|c|}{ Município } & $\mathrm{N}^{0}$ de indivíduos & $(\%)$ & \multicolumn{1}{c}{ Municííio } & $\mathrm{N}^{0}$ de indivíduos & $(\%)$ \\
\hline Araricá & 5 & $0,03 \%$ & Nova Hartz & 19 & $0,13 \%$ \\
Campo Bom & 239 & $1,62 \%$ & Novo Hamburgo & 1.338 & $9,07 \%$ \\
Canoas & 862 & $5,84 \%$ & Portão & 197 & $1,34 \%$ \\
Dois Irmãos & 153 & $1,04 \%$ & Porto Alegre & 1.725 & $11,69 \%$ \\
Estância Velha & 158 & $1,07 \%$ & São Leopoldo & 3.505 & $23,75 \%$ \\
Esteio & 821 & $5,56 \%$ & Sapiranga & 209 & $1,42 \%$ \\
Ivoti & 148 & $1,00 \%$ & Sapucaia do Sul & 1.248 & $8,46 \%$ \\
Nova Santa Rita & 22 & $0,15 \%$ & Outras cidades & 4.106 & $27,83 \%$ \\
\hline
\end{tabular}

Fonte: Elaborada pelos autores com base nos dados da pesquisa.

A cidade de Porto Alegre é o segundo município gaúcho que abriga o maior número de universitários da UNISINOS e juntamente com Novo Hamburgo, Sapucaia do Sul, Canoas, Esteio e São Leopoldo abriga 64,38\% de todos os discentes pesquisados no período.

No que se refere ao estado civil, tem-se que a amostra era composta em sua expressiva maioria por solteiros $(86 \%)$, seguido de casados (10\%). Dois por cento dos alunos não informaram seu estado civil e dois por cento eram divorciados ou separados. Já em termos de instrução dos pais, $23,97 \%$ possuíam o ensino fundamental incompleto e $25,08 \%$ o ensino médio. A Tabela 2 permite visualizar a distribuição completa para os diferentes níveis de formação.

Tabela 2 - Grau de Instrução dos pais dos alunos da UNISINOS ingressantes no período de 2005 a 2008

\begin{tabular}{|lcccc|}
\hline \multicolumn{1}{c}{ Grau de Instrução } & \multicolumn{2}{c}{ Pai } & \multicolumn{3}{c|}{ Mãe } \\
\cline { 2 - 5 } Analfabeto & $\begin{array}{c}N^{o} \text { de } \\
\text { indivíduos }\end{array}$ & $(\%)$ & $N^{o}$ de & $(\%)$ \\
individuos & \\
Ensino Fundamental Incompleto & 101 & $0,68 \%$ & 110 & $0,75 \%$ \\
Ensino Fundamental & 3.442 & $23,33 \%$ & 3.537 & $23,97 \%$ \\
Ensino Médio Incompleto & 1.701 & $11,53 \%$ & 2.070 & $14,03 \%$ \\
Ensino Médio & 1.145 & $7,76 \%$ & 1.346 & $9,12 \%$ \\
Ensino Superior Incompleto & 3.422 & $23,19 \%$ & 3.700 & $25,08 \%$ \\
Ensino Superior & 1.354 & $9,18 \%$ & 1.287 & $8,72 \%$ \\
Especialização & 1.566 & $10,61 \%$ & 1.804 & $12,23 \%$ \\
Mestrado & 336 & $2,28 \%$ & 471 & $3,19 \%$ \\
Doutorado & 122 & $0,83 \%$ & 145 & $0,98 \%$ \\
Pós-Doutorado & 45 & $0,30 \%$ & 40 & $0,27 \%$ \\
Livre Docência & 18 & $0,12 \%$ & 25 & $0,17 \%$ \\
Não responderam & 5 & $0,03 \%$ & 8 & $0,05 \%$ \\
\end{tabular}

Fonte: Elaborada pelos autores com base nos dados da pesquisa.

Com relação ao tipo de ensino médio (segundo grau ou equivalente) concluído pelos estudantes, apurou-se que 7.958 alunos, ou seja, 53,93\% da amostra, concluíram o ensino médio regular, através do qual o ensino mostra-se mais abrangente que os demais, possibilitando assim uma preparação mais específica para o vestibular. O técnico/ profissionalizante foi o segundo maior estrato, somando 2.389 alunos (16,19\% do total da amostra). Logo após tem-se o supletivo, que somou 1.959 universitários, isto é, $13,28 \%$ do total de pesquisados. E, em termos de tipo de escola cursada, $50 \%$ vieram de escolas públicas estaduais e $39 \%$ de escolas privadas. Seis por cento não responderam e os demais se distribuíram, de forma semelhante, entre escolas públicas municipais e federais.

Verificou-se que $82,14 \%$ dos alunos que ingressaram na UNISINOS no período analisado nunca haviam dado início a nenhum curso superior e que 43,23\% dos alunos responderam que haviam escolhido o curso de graduação por adequação as suas aptidões pessoais, enquanto somente $2,81 \%$ escolheram o curso pelo fato de proporcionar amplas possibilidades salariais. Isso mostra que os estudantes estão preocupados cada vez mais com a sua realização pessoal $(18,11 \%)$ em detrimento do prestígio social que a profissão pode lhe gerar $(2,17 \%)$.

Do total de estudantes da UNISINOS, 56,63\% exerciam algum tipo de atividade remunerada, 21\% contribuíam parcialmente com o sustento da família e $27,71 \%$, eram responsáveis pelo seu próprio sustento.

A fatia de renda mensal dominante situou-se entre três e cinco salários mínimos $(27,59 \%)$ e somente 3,67\% dos universitários afirmaram que a renda mensal de sua família era de vinte ou mais salários mínimos.

Analisando a distribuição de renda e os alunos evadidos, detectou-se que o estrato com maior número de alunos evadidos foi o de três a menos de cinco salários mínimos, com uma participação de evadidos de 14,67\%. No entanto, esse estrato é o que possuía o maior número de alunos (27,59\%). Assim, é necessário avaliar o valor relativo da evasão por faixa salarial. Essa avaliação permitiu verificar que a faixa com maior evasão relativa foi a de menor renda e que essa participação foi se reduzindo à medida que se amplia a renda, com exceção da última faixa de renda (mais de vinte salários mínimos), que sofre um pequeno aumento. Esses dados reforçam os resultados encontrados por outros pesquisadores, como evidenciado na introdução, de que faixas de menor renda apresentam 
maior probabilidade de evadir em função de dificuldades financeiras. A Tabela 3 permite avaliar essas informações.

Tabela 3 - Renda total mensal (em salários mínimos - s. m.) de acordo com a situação acadêmica dos alunos da UNISINOS ingressantes no período de 2005 a 2008

\begin{tabular}{|c|c|c|c|c|c|c|c|}
\hline \multirow{3}{*}{$\begin{array}{c}\text { Renda total mensal } \\
\text { (em salários mínimos - s.m. } \text {.) }\end{array}$} & \multirow{3}{*}{$\begin{array}{l}\mathrm{N}^{\mathrm{d}} \mathrm{de} \\
\text { Indivíduos }\end{array}$} & \multirow{3}{*}{$\%$} & \multicolumn{4}{|c|}{ Sittuação acadêmica } & \multirow{3}{*}{$\%$ Relativ } \\
\hline & & & \multicolumn{2}{|c|}{ Ativo } & \multicolumn{2}{|l|}{ Evadido } & \\
\hline & & & $\begin{array}{c}\mathrm{I}^{0} \mathrm{de} \\
\text { Indivíduos }\end{array}$ & $\%$ & $\begin{array}{l}\mathrm{N}^{0} \mathrm{de} \\
\text { Indivíduos }\end{array}$ & $\%$ & \\
\hline Até menos de dois s. m. & 896 & 6,07 & 367 & 2,49 & 529 & 3,59 & 59,06 \\
\hline De dois a menos de três s. m. & 2.225 & 15,08 & 957 & 6,49 & 1.268 & 8,59 & 56,99 \\
\hline De três a menos de cinco s. m. & 4.071 & 27,59 & 1.907 & 12,92 & 2.164 & 14,67 & 53,16 \\
\hline De cinco a menos de setes m. & 2.722 & 18,45 & 1.339 & 9,07 & 1.383 & 0,37 & 50,80 \\
\hline De sete a menos de dezs. m. & 1.706 & 11,56 & 924 & 6,26 & 782 & 5,30 & 45,85 \\
\hline De dez a menos de quinze s. m. & 1.217 & 8,25 & 715 & 4,85 & 502 & 3,40 & 41,24 \\
\hline De quinze a menos de vinte s. $m$ & 497 & 3,37 & 305 & 2,07 & 192 & 1,30 & 38,61 \\
\hline De vinte ou mais s. m. & 542 & 3,67 & 327 & 2,22 & 215 & 1,46 & 39,70 \\
\hline Não responderam & 879 & 5,96 & 438 & 2,97 & 441 & 2,99 & 50,15 \\
\hline Total & 14.755 & 100,00 & 7.279 & 49,33 & 7.476 & 50,67 & 50,67 \\
\hline
\end{tabular}

Fonte: Elaborada pelos autores com base nos dados da pesquisa.

Em termos de cursos de graduação, o mais frequentado pelos estudantes da UNISINOS no período analisado foi o curso de Administração de Empresas Noturno (11,55\%), que também apresentou o índice de evasão mais baixo (6,92\%). Por sua vez, o Superior Tecnológico em Gestão Financeira foi o curso que apresentou o menor número de alunos em relação ao total da amostra $(0,14 \%)$. A graduação Superior Tecnológico em Gastronomia Noturno foi a que obteve o maior porcentual de alunos evadidos em relação ao total do curso $(81,25 \%)$. Essas e outras informações relativas a essas questões podem ser observadas na Tabela 4.

Tabela 4 - Estudantes evadidos em relação ao total da amostra de cada curso dos alunos da UNISINOS ingressantes no período de 2005 a 2008

\begin{tabular}{|c|c|c|c|c|c|}
\hline Curso & $(\%)^{1}$ & $(\%)^{2}$ & Curso & $(\%)^{1}$ & $(\%)^{2}$ \\
\hline Adm. Empresas - GIL & $0,47 \%$ & $11,59 \%$ & Jornalismo Not. & $3,06 \%$ & $47,45 \%$ \\
\hline Adm. Empresas Diu. & $1,65 \%$ & $7,82 \%$ & Letr. Port./Alem. Lic. Diu. & $0,29 \%$ & $25,58 \%$ \\
\hline Adm. Empresas Not. & $11,55 \%$ & $6,92 \%$ & Letras Hab. Port./Inglês Lic. & $1,46 \%$ & $49,07 \%$ \\
\hline Arquitetura e Urbanismo Diu. & $1,00 \%$ & $37,84 \%$ & Letras Inglês Lic. & $0,52 \%$ & $49,35 \%$ \\
\hline Arquitetura e Urbanismo Not. & $2,59 \%$ & $45,03 \%$ & Letras Port./Esp. Lic. & $0,17 \%$ & $68,00 \%$ \\
\hline Biologia Bach. Diu. & $0,81 \%$ & $43,33 \%$ & Letras Português Lic. & $0,58 \%$ & $59,30 \%$ \\
\hline Biologia Lic. Diu. & $1,79 \%$ & $48,86 \%$ & Matemática Lic. & $1,84 \%$ & $51,84 \%$ \\
\hline Ciência da Computação & $2,80 \%$ & $46,49 \%$ & Nutrição Not. & $2,86 \%$ & $49,53 \%$ \\
\hline Ciências Contábeis & $2,24 \%$ & $47,88 \%$ & Ped. Gest Sup PrEdu Diu & $0,24 \%$ & $54,29 \%$ \\
\hline Ciências Econômicas & $1,03 \%$ & $40,13 \%$ & Ped. Sup PrEdu Not. & $0,73 \%$ & $59,26 \%$ \\
\hline Ciências Sociais Lic. & $0,24 \%$ & $55,56 \%$ & Pedag. Educ. Especial & $0,24 \%$ & $42,86 \%$ \\
\hline Comércio Exterior Diu. & $0,28 \%$ & $35,71 \%$ & Pedagogia do Trabalho & $0,23 \%$ & $64,71 \%$ \\
\hline Comércio Exterior Not. & $1,84 \%$ & $54,61 \%$ & Pedagogia Lic. Not. & $1,34 \%$ & $48,22 \%$ \\
\hline Comunicação Digital & $0,22 \%$ & $27,27 \%$ & Psicologia & $4,01 \%$ & $47,72 \%$ \\
\hline Design & $0,43 \%$ & $31,75 \%$ & Publicidade e Propaganda & $3,46 \%$ & $50,00 \%$ \\
\hline Direito Diu. & $2,22 \%$ & $44,34 \%$ & Realização Audiovisual & $0,53 \%$ & $26,92 \%$ \\
\hline Direito Not. & $7,41 \%$ & $50,64 \%$ & Recursos Humanos & $0,20 \%$ & $55,17 \%$ \\
\hline Educação Física & $0,38 \%$ & $48,21 \%$ & Relações Públicas Not. & $1,17 \%$ & $50,00 \%$ \\
\hline
\end{tabular}

\begin{tabular}{|llllll|}
\hline Educação Física Lic. Not. & $3,66 \%$ & $56,85 \%$ & Serviço Social & $0,54 \%$ & $58,75 \%$ \\
Enfermagem & $3,02 \%$ & $61,21 \%$ & Sup Form Esp. Gastr Not. & $0,32 \%$ & $61,70 \%$ \\
Eng. da Computação & $0,20 \%$ & $20,00 \%$ & Sup. For. Prod. Mús. Rock & $0,43 \%$ & $29,69 \%$ \\
Eng. de Alimentos & $0,81 \%$ & $47,90 \%$ & Sup. Tec. Anál. de Sistemas & $0,86 \%$ & $59,06 \%$ \\
Eng. de Produção Not. & $4,51 \%$ & $59,46 \%$ & Sup. Tec. Gastron. Diu. & $0,54 \%$ & $64,56 \%$ \\
Eng. Mecânica Not. & $4,80 \%$ & $56,78 \%$ & Sup. Tec. Gastron. Not. & $0,22 \%$ & $81,25 \%$ \\
Engenharia Civil Not. & $2,35 \%$ & $39,19 \%$ & Sup. Tec. Gest. Financ. & $0,14 \%$ & $33,33 \%$ \\
Engenharia Elétrica & $2,74 \%$ & $54,21 \%$ & Sup. Tec. Gestão Ambiental & $1,04 \%$ & $49,67 \%$ \\
Filosofia Lic. Not. & $0,53 \%$ & $52,56 \%$ & Sup. Tec. Logística & $0,75 \%$ & $49,55 \%$ \\
Física Lic. & $0,72 \%$ & $68,87 \%$ & Sup. Tec. Seg. Informação & $1,19 \%$ & $41,71 \%$ \\
Fisioterapia & $2,02 \%$ & $58,05 \%$ & Sup.Tec. em Jogos Digitais & $1,44 \%$ & $51,42 \%$ \\
Geologia Not. & $0,60 \%$ & $54,55 \%$ & Sup.Tec. Rec. Human. & $0,69 \%$ & $44,12 \%$ \\
História Lic. & $1,60 \%$ & $51,69 \%$ & & & \\
\hline
\end{tabular}

Fonte: Elaborada pelos autores com base nos dados da pesquisa.

Notas:

${ }^{1}$ Porcentual de alunos em relação ao total da amostra.

${ }^{2}$ Porcentual de evadidos em relação ao total do curso.

Também se constatou que os cursos diurnos foram os que apresentaram os menores índices de evasão em relação aos noturnos, consequência do fato de muitos universitários serem "trabalhadores-estudantes", o que dificulta a conciliação entre o trabalho, que é o meio necessário à sobrevivência, e os estudos, conforme já citado na introdução.

Em resumo, da totalidade da amostra, que somou 14.755 discentes com ingressos de 2005/2 a 2008/2, constatou-se que os estudantes, em sua maioria, tinham estas características: eram do sexo feminino, com maior volume de ingressos na universidade no período letivo de 2008/1; eram residentes na cidade de São Leopoldo, município onde se localiza a UNISINOS; eram solteiros; tinham o pai com grau de instrução do ensino fundamental incompleto, diferentemente da mãe, que possuía o ensino médio como grau de escolaridade; concluíram o ensino médio regular; frequentaram escola estadual durante o dia; não frequentaram curso pré-vestibular ou prestaram a prova em outros semestres; realizaram o concurso vestibular somente na UNISINOS; nunca haviam iniciado algum curso superior.

Relataram, ainda, que o motivo principal da escolha do curso no qual se inscreveram foi a adequação as suas aptidões pessoais; que exerciam algum tipo de atividade remunerada de 40 horas semanais ou mais, e que a renda total mensal, em salários mínimos, de todas as pessoas que residiam em suas casas eram de três até cinco salários mínimos. 
Fatores determinantes da evasão universitária: uma análise com alunos da UNISINOS

\subsection{Análise do Resultado Estatístico do Modelo Logístico}

Como esta pesquisa teve caráter exploratório, então acrescentarm-se todas as variáveis conforme mencionado na seção 3, a fim de que fosse possível avaliar o nível de significância de cada uma, retirando as não significantes até obter um modelo final que conteria os fatores determinantes da evasão. Destaca-se que se imputou ao aluno evadido o valor 1 (um) e 0 (zero) ao ativo. Logo, valores positivos do beta estariam indicando uma relação direta entre a variável em questão e a probabilidade de o aluno evadir.

Ainda, antes de realizar a estimação, avaliou-se a matriz de correlação entre as variáveis para verificar a existência de multicolinearidade. A quase totalidade dos dados apresentou correlações simples menores do que 0,30. Apenas duas foram superiores a 0,5, atingindo 0,82 e 0,80 , estado civil (solteiro ou casado) e tipo de escola (pública ou privada), respectivamente.

A falta de alguma(s) informação(ões) para determinados alunos fez com que eles fossem excluídos na análise de regressão. Assim, embora a amostra fosse composta por 14.755 alunos, o número de observações utilizadas no modelo logístico foi de 11.949 , dado o processo de exclusão.

Os resultados indicam que muitas das variáveis não exercem influência no modelo (pelo nível de significância). Dessa forma, eliminou-se a variável de menor significância reestimando sucessivamente o modelo até se obter apenas variáveis relevantes. Para níveis de significância inferior a 0,30 utilizou-se também como critério de exclusão o teste da Razão de Máxima Verossimilhança. Destaca-se que no modelo resultante desse procedimento deixaram-se as variáveis com níveis de significância iguais ou inferiores a 0,10. Uma vez obtida as variáveis significantes para a explicação da evasão, modelaram-se essas variáveis a fim de obter um melhor ajuste no modelo. Ao final desse procedimento obteve-se o resultado apresentado na Tabela 5.
Tabela 5 - Resultado do Modelo Logístico - somente variáveis significativas

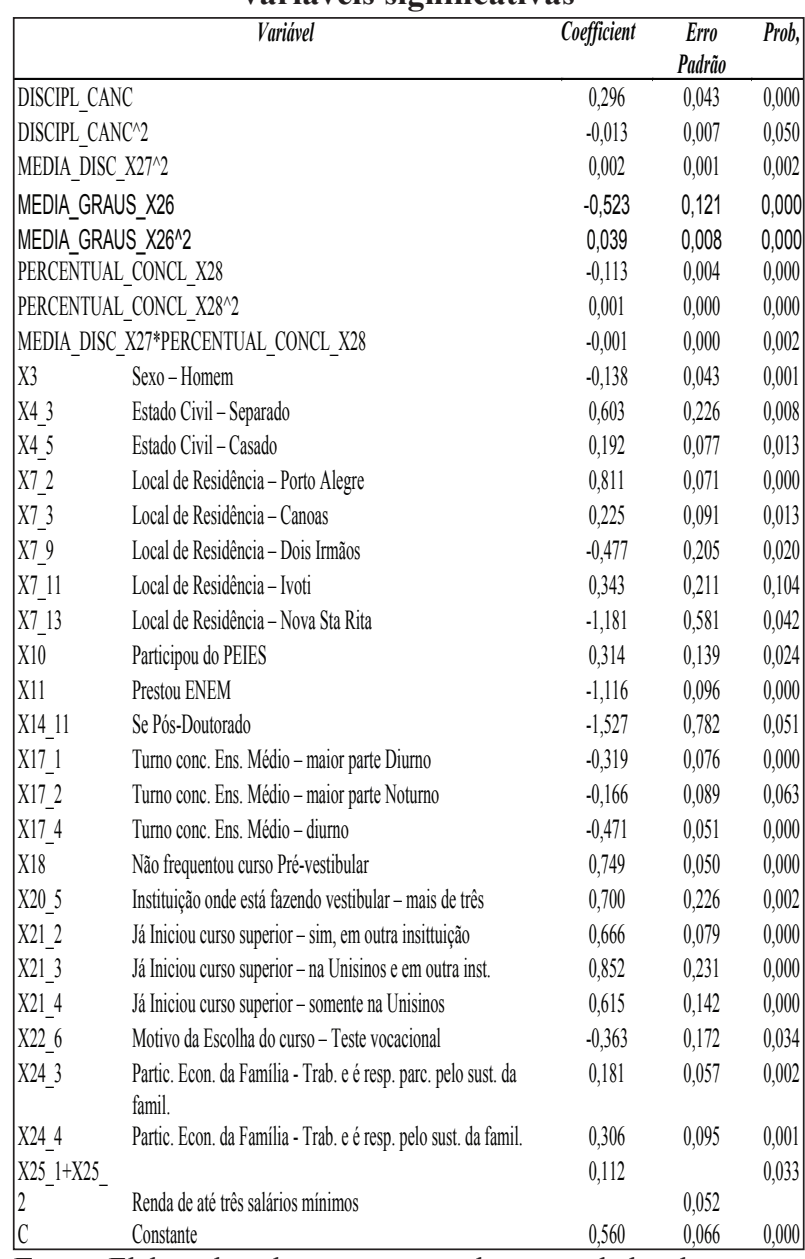

Fonte: Elaborada pelos autores com base nos dados da pesquisa.

Avaliando os resultados da Tabela 5, verifica-se que o aumento das disciplinas canceladas aumenta a probabilidade de evadir, porém o efeito se dá a taxas decrescentes. Já no que se refere à nota média e/ou porcentual de disciplinas cursadas, menoré a probabilidade de o aluno evadir, porém essa probabilidade se dá a taxas crescentes até o limite de nota e da conclusão do curso, respectivamente. Esses dois últimos resultados também fazem sentido, uma vez que alunos só terão, em média, elevados desempenhos se exercerem um esforço para tal, e esse esforço só será realizado se houver satisfação com o curso. No que se refere ao porcentual concluído do curso, alunos formandos só evadirão por fatores realmente sérios, o que não é comum de se verificar.

Outra variável interessante é a que decorre da interação entre nota média e porcentual concluído. Essa mostra 
que essas duas variáveis compõem uma adicional da explicação da razão para a permanência do aluno na universidade.

Também se verifica que os homens são mais propensos a evadir do que as mulheres. No que diz respeito ao estado civil, alunos casados ou separados apresentam maior probabilidade de evadir do que em outras situações de estado civil. Marginalmente, casados têm maior probabilidade de evadir.

É interessante notar que a evasão, em média, aumenta com a distância da residência do estudante ao campus (não levando em consideração os níveis de significância). No entanto, alunos moradores em Dois Irmãos, localidade afastada da sede da universidade, apresentam uma probabilidade menor de evasão do que a dos alunos que residem em São Leopoldo. O mesmo ocorre em Ivoti.

Estudantes que participaram do Programa de Ingresso ao Ensino Superior (PEIES) e os que não realizaram o Exame Nacional do Ensino Médio (ENEM) são mais propensos a evadir. Uma possível explicação para esses resultados é que o PEIES é uma seleção realizada pela Universidade Federal de Santa Maria (UFSM) e a distância da residência desses alunos da UNISINOS é relevante para o cancelamento e, ao mesmo tempo, sendo uma seleção para uma universidade federal talvez a motivação do aluno não se coadune com uma instituição particular.

Quanto à instrução dos pais, a única variável significativa foi a de mãe com pós-doutorado. Os filhos desse grupo de pessoas evadem menos que os demais. Essas variáveis, considerando as interações feitas para se obter o modelo final, apresentam uma significância maior para aqueles pais com maior formação e o efeito é de redução da evasão. Isso evidencia a possibilidade de os pais com níveis mais elevados de educação formal poderem orientar melhor seus filhos na questão da escolha dos cursos de graduação e, ao mesmo tempo, poderem aportar um apoio maior nos estudos dos filhos, seja na faculdade seja nos níveis anteriores à graduação. É curioso que esse fator tenha sido determinante apenas no caso da mãe, o que também induz a pensar que a mãe participa mais da vida escolar dos filhos do que os pais.

Os que concluíram outro tipo de ensino médio, que não era o ensino médio regular, tinham mais chances de abandonar a universidade sem concluir nenhum curso. E, ainda, pela magnitude do coeficiente estimado observa-se que quem cursou o ensino médio diurno é o que apresenta a menor probabilidade de evadir, dada a consideração do tipo de ensino médio.

Os estudantes da UNISINOS que não frequentaram curso pré-vestibular foram os mais propensos a evadir se comparados àqueles que frequentaram. E os discentes que fizeram o vestibular em mais de três instituições foram os que apresentaram a maior probabilidade de evadir, diante dos que prestaram em três ou até menos de três instituições.

Alunos que já haviam iniciado e abandonado um curso superior apresentaram maior probabilidade de evasão do que aqueles que não haviam cancelado uma faculdade. Isso, de certa forma, deveria ser com sinal contrário caso se pensasse que o estudante poderia ter iniciado um curso que não tivesse lhe interessado, e aí tenha refletido e decidido por trocar de curso. Porém, essa talvez não tenha sido a razão da maioria que optou por cancelar o curso. Uma possibilidade seria a pouca formação de base que fez com que esses alunos trocassem de curso de forma mais intensa, porém não há diferença de média das notas para esses grupos de alunos.

Pela análise do resultado estatístico, verificou-se que os alunos que já haviam iniciado algum curso superior na UNISINOS e também em outra instituição foram os que apresentaram a maior probabilidade de sair da universidade sem serem diplomados, assim como aqueles cujo motivo principal da escolha do curso no qual se inscreveram foi o prestígio social da profissão.

Destaca-se que alunos que participaram de testes vocacionais apresentaram menor probabilidade de evadir, resultado que reforça a relevância dessa atividade nas universidades. Pode ser inclusive um argumento contra a retórica de custos, pois os custos da evasão para a universidade são significantes. No caso da universidade em questão, uma redução da evasão em um ponto 
porcentual geraria um incremento monetário aproximado de 269 mil reais, que pode ser considerado como custo de oportunidade da não ampliação de aproximadamente 74 alunos com teste vocacional.

No que tange à renda total mensal, constatou-se que aqueles cuja renda está situada até três salários mínimos possuíam maior probabilidade de evadir. Todas as demais faixas apresentavam sinal negativo, porém não foram significativas, o que reforça o argumento de que dificuldades financeiras estão associadas à evasão. Se forem computadas as variáveis relacionadas com níveis baixos de renda como desempenho na graduação, não ter feito curso pré-vestibular, ter necessidade de trabalhar para se sustentar na faculdade ou ainda ter de contribuir com o sustento da família, entre outros, então se pode concluir que a questão da renda é muito importante para a determinação da magnitude da evasão, pois ela se manifesta por várias dimensões.

O modelo estimado (apresentado na Tabela 5) permitiu um porcentual de acerto total de $76,76 \%$, o qual se distribuiu em um acerto de $81,74 \%$ daqueles alunos que não evadiram e $70,42 \%$ dos que evadiram, com uma probabilidade discriminante de 0,5. Esses dados podem ser observados na Tabela 6.

Tabela 6 - Avaliação dos resultados encontrados na estimação do modelo logístico (Probabilidade de evadir com discriminante entre estar ativo ou evadido de 0.5)

\begin{tabular}{|lccc|}
\hline & Dep=0 & Dep=1 & Total \\
\hline Probabilidade (Dep=1) $\leq C$ & 5.480 & 1.556 & 7.036 \\
Probabilidade (Dep=1)>C & 1.224 & 3.704 & 4.928 \\
Total & 6.704 & 5.260 & 11.964 \\
Correto & 5.480 & 3.704 & 9.184 \\
\% Correta & 81,74 & 70,42 & 76,76 \\
\% Incorreta & 18,26 & 29,58 & 23,24 \\
\hline
\end{tabular}

Fonte: Elaborada pelos autores com base nos dados da pesquisa.

Esse ajuste poderia ter sido significativamente melhorado se tivesse sido possível levar em consideração o fato de o aluno receber ou não bolsa de estudos ou algum tipo de auxílio. Como os dados trabalhados não continham essa informação, então muitos alunos com as características de um aluno que evadiria, poderia não tê-lo feito em função de possuir bolsa. Destaca-se que a UNISINOS possuía, no período de análise, aproximadamente um terço dos seus alunos com algum tipo de bolsa. A agregação dessa informação no modelo, crê-se, teria permitido ampliar o poder de explicação do modelo de forma significativa.

É interessante notar que em torno de $18 \%$ dos alunos com as características de um indivíduo que não evadiria acabaram por evadir da universidade. Essa informação é relevante, pois aproximadamente 1.200 alunos da amostra não teriam problemas relacionados a renda, distância do campus, dificuldade de aprendizado, e mesmo assim evadiram. Como no conceito de evasão não está sendo considerada a troca de curso, isso significa que esses alunos realmente abandonaram a universidade e evidencia a complexidade relacionada à questão da evasão.

Analisando os dados de forma individualizada, verificou-se que a variável Disciplinas Canceladas é a variável resumo para a gestão da evasão por parte da universidade. Ela não é uma variável causa em si, mas reflete outros elementos como dificuldades de aprendizado, disponibilidade de tempo para os estudos, capacidade financeira, para citar alguns, e apresenta uma relação crescente com a probabilidade de o aluno evadir. Controlando os demais fatores, na medida em que o número de disciplinas canceladas se eleva maior é a possibilidade de o aluno deixar a universidade sem concluir algum curso superior. O Gráfico 1 permite verificar a relação entre o número de

disciplinas canceladas e a probabilidade de evasão do aluno.

Gráfico 1 - Relação entre a quantidade de disciplinas canceladas e a probabilidade de evasão dos alunos da Universidade do Vale do Rio dos Sinos ingressantes no período de 2005 a 2008

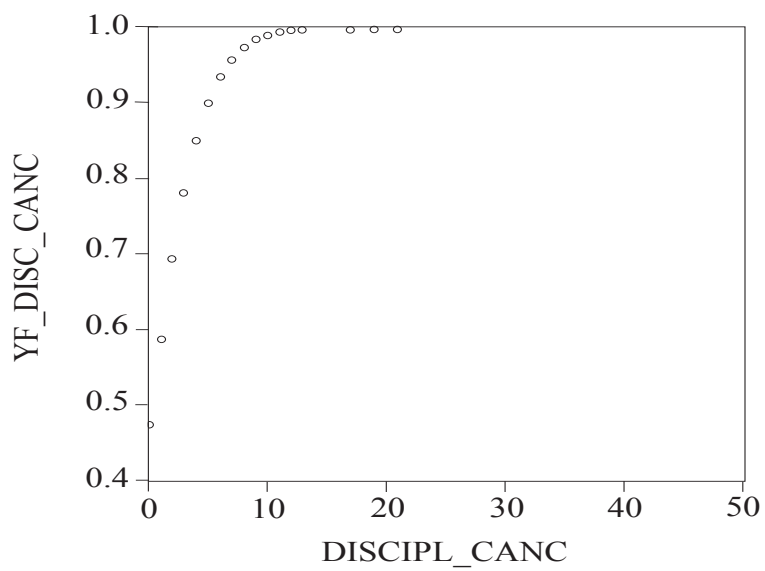

Fonte: Elaborado pelos autores com base nos dados da pesquisa.

Pela análise do Gráfico 1, verifica-se que alunos com cinco disciplinas canceladas já apresentam 90\% de probabilidade de evadir. Logo, como essa é uma variável de fácil acompanhamento pela instituição, os alunos que apresentem a terceira disciplina cancelada deveriam 
sofrer algum tipo de acompanhamento porque já possuem, aproximadamente, $50 \%$ de probabilidade de evadir.

De forma semelhante ao gráfico anterior, o Gráfico 2 também apresentou uma relação crescente entre a variável Disciplinas Reprovadas e a possibilidade de evasão dos alunos, em que quanto maior for o número de reprovações em disciplinas do currículo maior é a probabilidade de o aluno evadir.

Gráfico 2 - Relação entre a quantidade de disciplinas reprovadas e a probabilidade de evasão dos alunos

da Universidade do Vale do Rio dos Sinos ingressantes no período de 2005 a 2008

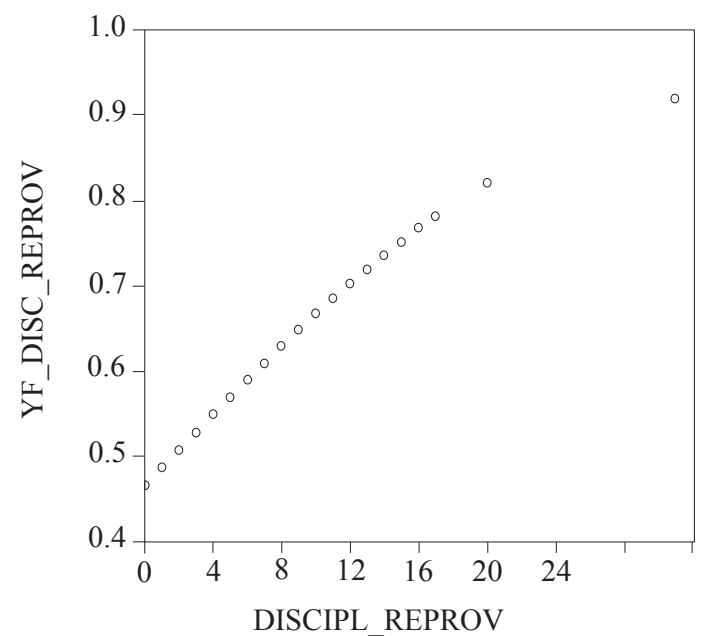

Fonte: Elaborado pelo autor com base nos dados da pesquisa.

Essa também poderia ser uma variável síntese para o acompanhamento da evasão pela instituição, pois alunos com quatro reprovações estão próximos de $50 \%$ de chance de evasão.

Em suma, a análise do modelo logístico permitiu encontrar 30 fatores determinantes da evasão com um nível de significância menor que 0,05 . Considerando que este foi um estudo exploratório, pela não existência de um modelo teórico que relacionaria a evasão com as variáveis trabalhadas, os resultados obtidos abrem a possibilidade de estudos qualitativos que possam explicar por que as variáveis definidas estão associadas com a evasão, bem como trabalhar um modelo teórico que possa explicar essa atitude dos estudantes.
Esta pesquisa analisou os fatores determinantes da evasão universitária dos alunos da Universidade do Vale do Rio dos Sinos, buscando identificar quais as variáveis que possuíam maior importância na decisão dos alunos em evadir ou não. Após a análise realizada, constatou-se que as variáveis mais relevantes (menor p-valor) para explicar a evasão foram as variáveis disciplinas canceladas, média de graus, porcentual de disciplinas concluída, município de residência, refletindo, provavelmente, a distância do campus, e conteria dois elementos: os custos de transportes e a facilidade de acesso, e o período em que os alunos realizaram o ensino médio. A distância, por sua vez, pode estar revelando a capacidade financeira da família e/ou as condições familiares e de serviços públicos de educação para o desenvolvimento da formação do aluno.

Verificou-se que existem diversos instrumentos que podem ser implementados pela universidade para reduzir a evasão. Um seria o incremento das atividades de orientação vocacional aos alunos, e o outro seria a criação de "oficinas" para o resgate de alunos com uma formação deficiente no ensino médio. Essas atividades, mesmo oferecidas gratuitamente pelas universidades particulares, poderiam gerar incremento nos resultados financeiros em razão da redução da evasão e dos custos decorrentes.

Como sugestão de trabalhos futuros, acredita-se que a replicação deste estudo em outras instituições de ensino superior poderia confirmar a relevância dos fatores aqui encontrados. Tendo em vista que este estudo foi exploratório, pesquisas qualitativas também poderiam contribuir para a formação de um quadro teórico a respeito da evasão.

\section{CONSIDERAÇÕES FINAIS}




\section{Apêndice - Variáveis e Dummies geradas para estimação do Modelo Logit}

DISCIPLINAS_CANCELADAS= Número de disciplinas canceladas.

DISCIPLINAS_REPROVADAS= Número de disciplinas reprovadas.

MÉDIA_DISCIPLINAS_CURSADAS = Número médio de disciplinas cursadas por semestre.

MÉDIA_GRAUS $=$ Desempenho médio do aluno (nota média).

PERCENTUAL_CONCLUÍDO = Número de disciplinas restantes para a formatura.

$\mathrm{X} 3$ = Sexo sendo: $\quad \mathrm{X} 3=1$ se homem e zero se mulher.

$\mathrm{X} 4$ = Estado Civil sendo:

X4 $1=1$ se solteiro e zero se outro;

$\mathrm{X} 4$ - 2 = 1 se divorciado e zero se outro;

X4_3 = 1 se separado e zero se outro;

$\mathrm{X} 4 \_4=1$ se viúvo e zero se outro;

$\mathrm{X} 4 \_5=1$ se casado e zero se outro;

X4_6 = 1 se não informado e zero se outro (variável subtraída e seu valor computado na constante).

$\mathrm{X} 5=$ Data de nascimento (não estimado).

X6 = Local de nascimento (não estimado).

$\mathrm{X} 7$ = Local onde reside sendo:

X7_1= 1 se São Leopoldo e zero se outro (variável subtraída e seu valor computado na constante);

$\mathrm{X} 7 \_2=1$ se Porto Alegre e zero se outro;

$\mathrm{X} 7 \_3=1$ se Canoas e zero se outro;

$\mathrm{X} 7^{-} 4=1$ se Esteio e zero se outro;

X7_5=1 se Sapucaia do Sul e zero se outro;

X7_6= 1se Novo Hamburgo e zero se outro;

X7_7 $=1$ se Araricá e zero se outro;

X7_8= 1 se Campo Bom e zero se outro;

X7_-9= 1 se Dois Irmãos e zero se outro;

$X 7{ }^{-} 10=1$ se Estância Velha e zero se outro;

$\mathrm{X} 7 \_11=1$ se Ivoti e zero se outro;

$X 7 \_12=1$ se Nova Hartz e zero se outro;

X7_13= 1 se Nova Santa Rita e zero se outro;

X7_14= 1 se Portão e zero se outro;

$\mathrm{X} 7 \_15=1$ se Sapiranga e zero se outro.

$\mathrm{X} 8{ }^{-}=$Nome da última Instituição de Ensino que frequentou (não estimado).

X9 = Deseja receber um atestado de comparecimento à prova (não estimado).

$\mathrm{X} 10$ = Participou do Programa de Ingresso ao Ensino Superior (PEIES) sendo:

$\mathrm{X} 10=1$ se sim e zero se não.

X11 = Prestou o Exame Nacional do Ensino Médio (ENEM) sendo:

$\mathrm{X} 11=1$ se sim e zero se não.

X12 = Além das aulas, para qual atividade teria o maior interesse em participar (não estimado).

X13 = Grau de Instrução do Pai sendo:

X13_1= 1 se analfabeto e zero se outro;

$\mathrm{X} 13 \_2=1$ se ensino fundamental incompleto e zero se outro;

X13_3= 1 se ensino fundamental e zero se outro;

$\mathrm{X} 13^{-} 4=1$ se ensino médio incompleto e zero se outro;

$\mathrm{X} 13{ }^{-} 5=1$ se ensino médio e zero se outro;

$\mathrm{X} 13$-6= 1 se superior incompleto e zero se outro;

$\mathrm{X} 13 \_7=1$ se superior e zero se outro;

X13_8=1 se especialização e zero se outro;

X13_9= 1 se mestrado e zero se outro;

$\mathrm{X} 13-10=1$ se doutorado e zero se outro;

$\mathrm{X} 13 \_11=1$ se pós-doutorado e zero se outro;

X13_12=1 se livre docência e zero se outro;

X13_13= grau de instrução não informado (variável subtraída e seu valor computado na constante).

X14 = Grau de Instrução da Mãe sendo:

X14_1= 1 se analfabeto e zero se outro; 
X14_2=1 se ensino fundamental incompleto e zero se outro;

$\mathrm{X} 14 \_3=1$ se ensino fundamental e zero se outro;

$\mathrm{X} 14 \_4=1$ se ensino médio incompleto e zero se outro;

$\mathrm{X} 14 \_5=1$ se ensino médio e zero se outro;

$\mathrm{X} 14 \_6=1$ se superior incompleto e zero se outro;

$\mathrm{X} 14 \_7=1$ se superior e zero se outro;

X14_8=1 se especialização e zero se outro;

X14_9=1 se mestrado e zero se outro;

$\mathrm{X} 14 \_10=1$ se doutorado e zero se outro;

$\mathrm{X} 14 \_11=1$ se pós-doutorado e zero se outro;

X14_12=1 se livre docência e zero se outro;

X14_13 = grau de instrução não informado (variável subtraída e seu valor computado na constante).

$\mathrm{X} 15$ = Tipo de ensino médio concluído sendo:

$\mathrm{X} 15 \_1=1$ se atual ensino médio e zero se outro;

$\mathrm{X} 15 \_2=1$ se magistério e zero se outro;

X15_3=1 se PPT/científico e zero se outro;

$\mathrm{X} 15 \_4=1$ se supletivo e zero se outro;

$\mathrm{X} 15$ 5 $=1$ se técnico/profissionalizante e zero se outro;

$\mathrm{X} 15$ 6 6 = 1 se outro e zero se outro

X15_7= tipo de ensino médio concluído não informado (variável subtraída e seu valor computado na constante).

$\mathrm{X} 16$ = Tipo de escola onde frequentou o ensino médio sendo:

X16_1= 1 se estadual e zero se outro;

X16_2=1 se federal e zero se outro;

X16_3=1 se municipal e zero se outro;

X16_4= 1 se não informado e zero se outro (variável subtraída e seu valor computado na constante);

X16_5 $=1$ se privado e zero se outro.

$\mathrm{X} 17=$ Turno que concluiu o ensino médio sendo:

$\mathrm{X} 17 \_1=1$ se a maior parte no diurno e zero se outro;

X17_2=1 se a maior parte no noturno e zero se outro;

$\mathrm{X} 17^{-} 3=1$ se outro turno e zero se outro (variável subtraída e seu valor computado na constante);

$\mathrm{X} 17 \_4=1$ se todo diurno e zero se outro;

$\mathrm{X} 17$ 5 $=1$ se todo noturno e zero se outro.

$\mathrm{X} 18$ = Frequentou curso pré-vestibular no decorrer do último ano sendo:

$\mathrm{X} 18=1$ se não e zero se sim.

$\mathrm{X} 19=$ Semestres anteriores a este em que fez vestibular sendo:

X19_1= 1 se nenhum e zero se outro (variável subtraída e seu valor computado na constante);

$\mathrm{X} 19-2=1$ se um e zero se outro;

$\mathrm{X} 19 \_3=1$ se dois e zero se outro;

X19_4= 1 se três e zero se outro;

X19_5= 1 se mais de três e zero se outro.

X20 = Instituições onde está fazendo ou fará vestibular sendo:

X20_1= 1 se apenas na Unisinos e zero se outro (variável subtraída e seu valor computado na constante);

$\mathrm{X} 20 \_2=1$ se em mais uma e zero se outro;

$\mathrm{X} 20 \_3=1$ se em mais duas e zero se outro;

$\mathrm{X} 20 \_4=1$ se em mais três e zero se outro;

X20_5=1 se em mais de três e zero se outro.

X21 = Já iniciou algum curso superior sendo:

X21_1=1 se não e zero se outro (variável subtraída e seu valor computado na constante);

X21_2= 1 se sim, em outra instituição e zero se outro;

X21_3=1 se sim, na Unisinos e em outra instituição e zero se outro;

X21_4= 1 se sim, somente na Unisinos e zero se outro.

$\mathrm{X} 22$ = Motivo principal da escolha do curso sendo:

X22_1= 1 se adequação às minhas aptidões pessoais e zero se outro;

$\mathrm{X} 22 \_2=1$ se amplas possibilidades salariais e zero se outro;

$\mathrm{X} 22 \_3=1$ se forte influência da família e zero se outro;

X22_4= 1 se mercado de trabalho e zero se outro; 
X22_5=1 se outro motivo e zero se outro (variável subtraída e seu valor computado na constante);

$\mathrm{X} 22^{-} 6=1$ se por indicação no teste vocacional e zero se outro;

$\mathrm{X} 22^{-} 7=1$ se possibilidade de contribuir com a sociedade e zero se outro;

$\mathrm{X} 22 \_8=1$ se possibilidade de realização pessoal e zero se outro;

X22_9= 1 se pouca concorrência nas vagas e zero se outro;

$\mathrm{X} 22 \_10=1$ se prestígio social da profissão e zero se outro.

$\mathrm{X} 23$ = Exerce algum tipo de atividade remunerada sendo:

X23_1= 1 se não e zero se outro (variável subtraída e seu valor computado na constante);

X23_2= 1 se não, mas pretendo trabalhar durante o curso e zero se outro;

$\mathrm{X} 23 \_3=1$ : se sim, de 40 horas semanais ou mais e zero se outro;

X23_4= 1 se sim, até 20 horas semanais e zero se outro;

$\mathrm{X} 23{ }^{-} 5=1$ se sim, de 21 a 39 horas semanais e zero se outro;

$\mathrm{X} 23{ }^{-} 6=1$ se sim, eventualmente e zero se outro;

$\mathrm{X} 23{ }^{-} 7=1$ se sim, mas não pretendo trabalhar durante o curso e zero se outro.

X24 = Participação na vida econômica da família sendo:

X24_1= 1 se não trabalho e zero se outro (variável subtraída e seu valor computado na constante);

X24_2=1 se trabalho e sou responsável pelo próprio sustento e zero se outro;

X24_3= 1 se trabalho, sou responsável pelo próprio sustento e contribuo parcialmente para o sustento da família ou de outras pessoas e zero se outro;

X24_4= 1 se trabalho e sou o principal responsável pelo sustento da família e zero se outro.

X25 = Renda familiar, em salários mínimos, sendo:

X25_1= 1 se até menos de dois salários mínimos e zero se outro;

$\mathrm{X} 25 \_2=1$ se de dois até menos de três salários mínimos e zero se outro;

X25_3 $=1$ se de três até menos de cinco salários mínimos e zero se outro;

$\mathrm{X} 25$ - $4=1$ se de cinco até menos de sete salários mínimos e zero se outro;

$\mathrm{X} 25-5=1$ se de sete até menos de dez salários mínimos e zero se outro;

X25_6= 1 se de dez até menos de quinze salários mínimos e zero se outro;

X25_7= 1 se de quinze até menos de vinte salários mínimos e zero se outro;

X25_8= 1 se vinte ou mais salários mínimos e zero se outro (variável subtraída e seu valor computado na constante)

$\mathrm{C}=$ efeito médio das variáveis dummies que serviram de base no modelo.

Cursos

- ADMHABADM EMPRESAS NOT;

- ADMHAB COMÉRCIO EXTERIOR DIU;

- ADMHAB COM EXTERIOR NOT;

- ARQUITETURA URBANISMO DIU;

- ARQUITETURA URBANISMO NOT;

- BAC CIÊNCIA DA COMPUTAÇÃO;

- BAC EM ADMI. - NOTURNO;

- BAC EM ADMI. - DIURNO;

- BACH EM ADMI. - DIURNO;

- BACH EM ADMI. - NOTURNO;

- BACH EM DESIGN - HAB DESIGN;

- BACH EM EDUCAÇÃO FÍSICA;

- BACH SISTEMAS DE INFORMAÇAO;

- BACHARELADO EM ADMI.;

- CIÊNCIAS BIOLÓGICAS BAC DIU;

- CIÊNCIAS BIOLÓGICAS LIC DIU;

- CIÊNCIAS CONTÁBEIS;

- CIÊNCIAS ECONÔMICAS;

- CIÊNCIAS SOCIAIS LICENCIATURA;

- COM SOCRELPÚBNOT;

- COMUNICAÇÃO DIGITAL;

- COM SOCIAL JORNALISMO NOT;

- COM SOCIAL PUB E PRO;

- COM SOCIAL REALIZAÇÃO AUD;
- ENGHAB ENGENHARIA CIVIL NOT;

- FILOSOFIA LICENCIATURA NOT;

- FISIOTERAPIA;

- GEOLOGIA NOTURNO;

- HISTÓRIA LICENCIATURA;

- LETRAS HAB INGLÊS LIC;

- LETRAS HAB POR/ESPLIC;

- LETRAS HAB PORT/ALEMLIC DIU;

- LETRAS HAB PORT/INGLÊS LIC;

- LETRAS HAB PORTUGUÊS LIC;

- LIC EM PEDAGOGIA NOTURNO;

- LICENCIATURA EM FÍSICA;

- LICENCIATURA EM MATEMÁTICA;

- NUTRIÇÃO NOTURNO;

- PEDAGOGIA EDUCAÇÃO ESPECIAL;

- PEDAGOGIA GESTSUP PR EDU DIU;

- P EDAGOGIA GESTSUP PR EDU NOT;

- PEDAGOGIA - PEDAG DO TRABALHO;

- PSICOLOGIA;

- SERVIÇO SOCIAL;

- SUP FORM ESPEC GASTR DIU;

- SUP FORM ESPEC GASTR NOT;

- SUP DE TECNOLOGIA EM LOGÍSTICA;

- SUPFORMPROD MÚSICOS DE ROCK; 
- DIREITO BAC CIÊNJURSOC DIU;

- DIREITO BAC CIÊNJURSOCNOT;

- EDUCAÇÃO FÍSICA LICNOT;

- ENFERMAGEM;

- ENG ÁREA ELETRHABENGELÉTR;

- ENG COMPHABCOMPUT APL ENG;

- ENGHABENGMECÂNICA NOT;

- ENGHABENG PRODUÇÃO MEC NOT;

- ENGHAB ENGENHARIA ALIMENTOS;

\section{REFERÊNCIAS}

ALMEIDA, Edson Pacheco; VELOSO, Tereza Christina M. A. Evasão nos cursos de graduação da Universidade Federal de Mato Grosso, campus universitário de Cuiabá: Um processo de exclusão. Cuiabá: UFMT. 2002.

BAGGI, Cristiane Aparecida dos Santos; LOPES, Doraci Alves. Evasão e Avaliação Institucional no Ensino Superior: uma discussão bibliográfica. Avaliação, v. 16, n. 2, p. 355-374, jul. 2011.

BARDAGI, Marucia; HUTZ, Claudio Simon. Evasão universitária e serviços de apoio ao estudante: uma breve revisão da literatura brasileira. Psicologia Revista, v. 14 (2), p. 279301, nov. 2005.

BORGES, Priscilla. MEC e universidades estudam planos para combater evasão. Último Segundo/Educação. IG Brasília, 11/02/2012.

Disponível em: <http://ultimosegundo. ig.com.br/educacao/mec-e-universidadesestudam-planos-para-combater-evasao/ n1597622390779.html>. Acesso em: 18 mar. 2013.

DEKE, J. A. Study of the impact of public school spending on post secondary educational attainment using statewide school district refinancing in Kansas. Economics of Education Review, v. 22, n. 3, p. 275-284, Jun. 2003.

DIAS, Elaine Dal Mas. A dúvida da continuidade dos estudos universitários: uma questão adolescente. Taubaté: Cabral, 1997.
- SUPTECGEST RECURSOS HUMANOS

- SUPTEC GASTRONOMIA - DIURNO;

- SUPTEC GASTRONOMIA- NOTURNO;

- SUPTEC GESTÃO FINANCEIRA;

- SUPTEC SEGURANÇA INFORMAÇÃO;

- SUPTEC EM JOGOS DIGITAIS;

- SUPERIORTEC GESTÃO AMBIENTAL.

GARNER, Richard. More than $\mathbf{1 0 0 . 0 0 0}$ students drop out of university after first year. Londres, 26 julho 2007. Disponível em: $<\underline{\text { http:// }}$ www.independent.co.uk/news/education/ education-news/more-than-100000-studentsdrop-out-of-university-after-first-year-458758. html>. Acesso em: 09 maio 2009. Texto postado no The Independent's, no link Education.

SILVA FILHO, Roberto L. Lobo; MOTEJUNAS, Paulo Roberto; HIPÓLITO, Oscar; LOBO, Maria Beatriz de C. M. A evasão no ensino superior brasileiro. Cadernos de Pesquisa, v. 37, n. 132, p. 641 - 659, set./dez. 2007.

MACGREGOR, Karen. South Africa: Student drop-out rates alarming. University World News, Londres, ed. 3, out. 2007. Disponível em: $<$ http://www.universityworldnews.com/article. php? story $=20071025102245380>$. Acesso em: 09 maio 2009.

MAJZUB, Rohaty; RAIS, Maisarah Muhammad. Teachers' and parents' perception on effective strategies for dropout prevention. Procedia Social and Behavioral Sciences. vol. 9, p. 10361041, 2010.

NASSAR, Silvia M; NETO, Eugênio R; CATAPAN, Araci H; PIRES, Maria M. S. Inteligência Computacional aplicada a Gestão Universitária: Evasão Discente. In: IV Colóquio Internacional Sobre Gestão Universitária na América do Sul, 2004, Florianópolis. Anais eletrônicos do IV Colóquio Internacional sobre Gestão Universitária na América do Sul. Florianópolis: Universidade Federal de 
Santa Catarina, 2004. Disponível em: <http:// www.inpeau.ufsc.br/wp/v1/detalhe-artigos. php?id=407>. Acesso em: 24 mar. 2008.

PEREIRA, J. T. V. Uma contribuição para o entendimento da evasão: Um estudo de caso. São Paulo: UNICAMP, 1995.

REIS, Mônica. Evasão: o tabu da universidade. 2007. Disponível em: <http://www. olharvirtual.ufrj.br/2006/index.php?id edicao $=183 \& \operatorname{codigo}=1>$. Acesso em: 09 maio 2009.

RISTOFF, Dilvo I.Expressão sobre a graduação: Considerações sobre evasão. Maringá: UEM, 1997.

SILVA, Renato. Deserción: Competitividad ó Gestion. Revista Lasallista de Investigación. Colômbia, v. 2, p. 64-69, 2006.

STRATTON, Leslie S.; O’TOOLE, Dennis M.; WETZEL, JAMES N. A multinomial logit model of college stopout and dropout behavior. Economics of Education Review. Volume 27, Issue 3, p. 319-331, June 2008,

THEÓPHILO, Carlos R; MORAES, Júlia O. Evasão no Ensino Superior: Estudo dos fatores causadores da evasão no Curso de Ciências Contábeis da Universidade Estadual de Montes Claros - UNIMONTES. Montes Claros: UNIMONTES, 2005.

VIEIRA, Edemundo R; FRIGO, Lerci P. Evasão dos cursos de graduação da UFRGS em 1985, 1986 e 1987. 1. Ed. Porto Alegre: UFRGS, 1991.

VITELLI, Ricardo Ferreira. Evasão em cursos de graduação: fatores intervenientes no Fenômeno. Segunda Conferencia latino americana sobre el abandono en la educación - II CLABES, 2012. 